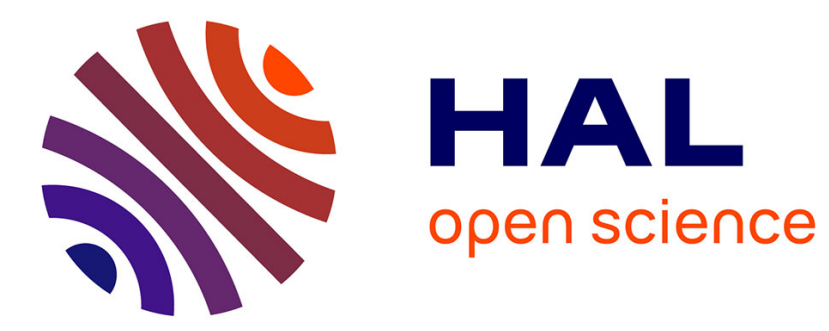

\title{
Position and mass determination of multiple particles using cantilever based mass sensors
}

\author{
Soren Dohn, Silvan Schmid, Fabien Amiot, Anja Boisen
}

\section{To cite this version:}

Soren Dohn, Silvan Schmid, Fabien Amiot, Anja Boisen. Position and mass determination of multiple particles using cantilever based mass sensors. Applied Physics Letters, 2010, 97 (4), 14p. 10.1063/1.3473761 . hal-00520914

\section{HAL Id: hal-00520914 \\ https://hal.science/hal-00520914}

Submitted on 24 Sep 2010

HAL is a multi-disciplinary open access archive for the deposit and dissemination of scientific research documents, whether they are published or not. The documents may come from teaching and research institutions in France or abroad, or from public or private research centers.
L'archive ouverte pluridisciplinaire HAL, est destinée au dépôt et à la diffusion de documents scientifiques de niveau recherche, publiés ou non, émanant des établissements d'enseignement et de recherche français ou étrangers, des laboratoires publics ou privés. 


\section{Position and mass determination of multiple particles using cantilever based mass sensors}

Søren Dohn ${ }^{\mathrm{a}, 1}$ Silvan Schmid ${ }^{\mathrm{a}}$ Fabien Amiot ${ }^{\mathrm{b}}$ Anja Boisen ${ }^{\mathrm{a}}$

a Department of Micro- and Nanotechnology, Technical University of Denmark, DTU Nanotech, Building 345 East, DK-2800 Kongens Lyngby, Denmark

${ }^{\mathrm{b}}$ FEMTO-ST Institute, CNRS-UMR 6174/UFC/ENSMM/UTBM, 24 chemin de l'Épitaphe, F-25030 Besançon, France

Using micro-scale cantilevers as sensitive mass sensors was proposed in 1995 [1,2]. Since then, cantilever based mass sensors have been shown to have the sensitivity to measure single cells and large molecules [3-5]. Recently, micro-beam based sensors have been used as mass spectrometers detecting single molecules [6].

With cantilever based mass sensors either a single added mass [7], a multitude of added masses creating a homogeneous layer

[5], or multiple single particle adsorption events can be detected [6]. When measuring the homogeneous adsorption of a multitude of added masses it is assumed these are homogeneously spread 
over the surface in order to quantify the response and calculate the corresponding additional mass. However, for the detection of individual adsorbed masses, the mass response of the cantilever will change with the actual position of the added mass since the vibration velocity of the cantilever surface varies with position [8]. Thus, the position of the mass adsorption needs to be controlled and is usually set to be at the cantilever tip or at a nodal point [9]. But for real-life measurements, the exact position of an added mass can not be controlled and none of the above methods are suitable for quantitative single or multiple particle detection.

We have previously shown that it is possible to find the position and the mass of a single particle adhering to a micro-cantilever by measuring the frequency response of higher order bending modes. [10] The drawback of the technique was, that it was possible to do measurements if only a single particle was added to the cantilever in between successive measurements. In this work we demonstrate, that the theory can be extended to detection of multiple particles with different masses. The theory is applied to measurements on several micro-cantilevers each loaded with multiple micro-particles of the same kind. Both, the positions and the mass of the individual particles are calculated and compared 
to the measured values of the positions and the theoretical mass of the micro-particles.

In our previous work, the resonant frequency change of a cantilever due to a small single mass, $\Delta m$ positioned at $z_{\Delta m}$, has been derived by equalizing the kinetic with the strain energy at resonance [10]. Considering $P$ populations of particles with a mass $\Delta m_{p}$ and $M_{p}$ particles at positions $z_{\Delta m_{p}, i}, i \in\left\{1 \cdots M_{p}\right\}$ the kinetic energy of a cantilever vibrating at a resonant frequency $\omega_{n, \Delta m}$ is

$$
E_{\mathrm{kin}, \sum \Delta m}=\frac{1}{2} a_{n}^{2} \omega_{n, \Delta m}^{2} \sum_{p=1}^{P} \Delta m_{p} \sum_{i=1}^{M_{p}} U_{n}^{2}\left(z_{\Delta m_{p}, i}\right)
$$

where $U_{n}$ is the mode shape of vibration and $a_{n}^{2}$ is the amplitude of the $n^{\text {th }}$ mode. Assuming that the added particles do not alter the mode shape of the cantilever, the strain energy does not change with the particle adsorption and is thus equal to the kinetic energy without particles. At resonance, the kinetic and the strain energy are set equal and the resonant frequency for a loaded cantilever becomes

$$
\omega_{n, \Delta m}^{2}=\omega_{n}^{2}\left(1+\sum_{p=1}^{P} \frac{\Delta m_{p}}{m_{0}} \sum_{i=1}^{M_{p}} U_{n}^{2}\left(z_{\Delta m_{p}, i}\right)\right)^{-1}
$$

where $m_{0}=w L t \rho$ is the mass of the cantilever. 
For the calculation of the positions and the mass of the attached particles based on the resonant frequency changes Eq.(2) is recast

$$
\mathbf{U} \rho \bar{d}=\overline{R_{\omega}}
$$

Using $N$ measured modes the matrix $\mathbf{U}$ of $N \times P$ elements and the vector $\bar{R}_{\omega}$ of $N$-elements are defined as

$$
\mathbf{U}=\left[\overline{u_{1}}, \overline{u_{2}}, \cdots, \overline{u_{P}}\right], \overline{u_{p}}=\left[\begin{array}{c}
\sum_{i=1}^{M_{p}} U_{1}^{2}\left(z_{\Delta m_{p}, i}\right) \\
\sum_{i=1}^{M_{p}} U_{2}^{2}\left(z_{\Delta m_{p}, i}\right) \\
\vdots \\
\sum_{i=1}^{M_{p}} U_{N}^{2}\left(z_{\Delta m_{p}, i}\right)
\end{array}\right], \overline{R_{\omega}}=\left[\begin{array}{c}
\frac{\omega_{1}^{2}}{\omega_{1, \Delta m}^{2}}-1 \\
\frac{\omega_{2}^{2}}{\omega_{2, \Delta m}^{2}}-1 \\
\vdots \\
\frac{\omega_{N}^{2}}{\omega_{N, \Delta m}^{2}}-1
\end{array}\right]
$$

$\bar{d}$ is the $P$-elements unitary vector and $\rho$ the scale factor so that

$$
\rho \bar{d}=\left[\begin{array}{c}
\frac{\Delta m_{1}}{m_{0}} \\
\frac{\Delta m_{2}}{m_{0}} \\
\vdots \\
\frac{\Delta m_{P}}{m_{0}}
\end{array}\right]
$$

The problem is then to find the positions $z_{\Delta m_{p}, i}$ and the relative 
mass changes satisfying Eq.(3). For a given positions set, the optimal $\bar{d}$ is obtained by solving

$$
\mathbf{U} \bar{d}=\frac{\overline{R_{\omega}}}{\left\|R_{\omega}\right\|}=\widetilde{R}
$$

in a least square sense: $\overline{d_{s o l}}=\left(\mathbf{U}^{\dagger} \mathbf{U}\right)^{-1}\left(\mathbf{U}^{\dagger} \widetilde{R}\right)$, where $\star^{\dagger}$ denotes the transpose of $\star$. Denoting the vector

$$
\bar{g}=\mathbf{U} \overline{d_{s o l}}-\widetilde{R}
$$

and minimizing $\chi^{2}=\bar{g}^{\dagger} \bar{g}$ with respect to the positions, the most likely positions of the attached particles can be located. The nonlinear minimization is performed under Matlab ${ }^{\circledR}$ using a NelderMead Simplex algorithm [11] and an initial guess found by a crude mesh calculation of $100^{M_{p}}$ positions. From the calculated positions, the scale factor is then calculated and the relative mass change of the individual particles obtained from Eq.(5) and:

$$
\rho=\frac{\left\|\overline{R_{\omega}}\right\|}{\left\|\mathbf{U} \overline{d_{\text {sol }}}\right\|} .
$$

The micro-cantilevers used in the experiment was fabricated from PECVD SiN having a thickness $t=850 \mathrm{~nm}$ deposited on a standard Si 4" wafer. The cantilevers are defined using photolithography followed by RIE giving cantilevers with a length $L=100 \mu \mathrm{m}$ 
and width $w=20 \mu \mathrm{m}$. The cantilevers are released by a $\mathrm{KOH}$ etch at $80{ }^{\circ} \mathrm{C}$ for $180 \mathrm{~min}$, and are subsequently coated with $20 \mathrm{~nm}$ of gold to improve their reflectivity. The total mass of the cantilevers are estimated to be $m_{0}=5.9 \mathrm{ng}$ with an estimated accuracy of $\pm 5 \%$ arising mainly from the uncertainty in the thickness of the gold layer $\left(\rho_{S i N}=3.0 \mathrm{~g} / \mathrm{cm}^{3}, \rho_{A u}=19.3 \mathrm{~g} / \mathrm{cm}^{3}\right)$. To actuate the cantilevers the cantilever chip is driven by a piezoactuator placed at the chip fixation.

Two different kinds of micro-particles are used in the experiments. Commercially available polystyrene microbeads (Polyscience, Polybead ${ }^{\circledR}, \rho_{p s}=1.05 \mathrm{~g} / \mathrm{cm}^{3}$ ) with a diameter of $2.0 \mu \mathrm{m}$ and magnetic microbeads (Invitrogen Dynabeads ${ }^{\circledR}$ M-280, $\rho_{M-280}=$ $1.3 \mathrm{~g} / \mathrm{cm}^{3}$ ) with a diameter of $2.8 \mu \mathrm{m}$. They were chosen to have different masses and the theoretical values are $4.4 \mathrm{pg}$ and $14.9 \mathrm{pg}$. The particles were positioned on the cantilever using an etched tungsten tip with a tip diameter of roughly $1 \mu \mathrm{m}$ mounted on a precision XYZ-stage under an optical microscope. An optical image of a cantilever loaded with 2 Dynabeads ${ }^{\circledR}$ is shown in Fig. 1.

The resonant frequencies of the first 5 to 7 bending modes have been measured with a laser-Doppler vibrometer (Polytec MSA- 
500) in vacuum (quality factor $>1000$ ) before and after loading the particles. A plot of the obtained relative changes in resonant frequencies for the first 5-7 bending modes of the cantilevers loaded with 2-3 Dynabeads ${ }^{\circledR}$ is shown in Fig. 2. The change in resonant frequency is between $0.03 \%$ and $0.83 \%$ depending on the number and the position of the particles.

It should be highlighted that the proposed identification procedure can be tailored to account for some a priori knowledge on the particles to be measured. If $M$ particles are to be measured, setting $P=1$ and $M_{1}=M$ imposes the same mass for all the particles. Another option is to set $P=M$ and $M_{p}=1 \forall p$ so that all particles are allowed to have different masses. Using Dynabeads ${ }^{\circledR}$ and allowing the particles to have different masses ( $\left.P=M, M_{p}=1\right)$, the calculated positions of the individual particles on the cantilevers, $z_{\Delta m_{1}, i}$ are plotted as a function of the positions measured using an optical microscope in Fig. 3. Excellent agreement is observed between the measured and the calculated positions for the experiments using both Dynabeads ${ }^{\circledR}$ and the lighter Polybeads ${ }^{\circledR}$. Based on all measurement the calculated mass-ratio of the particles to the cantilever is calculated and to quantify the error in the calculated position, the root- 


\begin{tabular}{lccccc} 
Beads & $\frac{\Delta m_{\text {actual }} \cdot 10^{3}}{m_{0}}$ & $\frac{\Delta m_{P=1}}{m_{0}} \cdot 10^{3}$ & $\frac{\widetilde{\Delta z}_{P=1}}{L}$ & $\frac{\Delta m_{P=M}}{m_{0}} \cdot 10^{3}$ & $\frac{\widetilde{\Delta z}_{P=M}}{L}$ \\
\hline Dynabeads ${ }^{\circledR}$ & $2.5 \pm 0.13$ & $2.5 \pm 0.37$ & 0.011 & $2.5 \pm 0.74$ & 0.012 \\
Polybeads ${ }^{\circledR}$ & $0.75 \pm 0.04$ & $0.85 \pm 0.08$ & 0.017 & $0.78 \pm 0.22$ & 0.018 \\
Table 1 & & & & &
\end{tabular}

Comparison of the particle-cantilever mass ratio obtained from the calculated positions shown in Fig. 3 to the actual values together with the root-mean-square value of the difference between the calculated and measured positions, $\widetilde{\Delta z}$.

mean-square value of the difference in calculated and measured position is calculated, $\widetilde{\Delta z}$. The mass-ratio and $\widetilde{\Delta z}$ are listed in table 1 for the cases of $P=1$ and $P=M$ for both kinds of particles. For both kinds of particles the agreement between the calculated mass-ratio and the theoretical value is good and within the uncertainty of the theoretical mass.

The uncertainty in the calculated mass-ratio and position increases for both kinds of particles when allowing the particles to have different masses. The effect is biggest for the lighter Polybeads ${ }^{\circledR}$ and is most likely due to the smaller changes in the measured resonant frequencies (an average relative frequency shift of only $0.11 \%$ were obtained using the Polybeads ${ }^{\circledR}$ compared to the $0.30 \%$ obtained with the Dynabeads $\left.{ }^{\circledR}\right)$. Because the measured frequency shifts are smaller they are more sensitive to errors coming from temperature changes and cantilever 
imperfections. The used cantilevers have an underetched anchor plate which alters the mode shapes compared to a perfect clamping assumed in the calculations. Furthermore, the measurements have been performed at ambient temperature without controlling it. Thus, the accuracy of the position and mass determination of particles can be improved first by increasing the sensitivity by increasing the mass ratio, that is using lighter cantilevers, second get rid of the imperfect cantilever clamping, that is using a different fabrication process for the cantilevers, and third by measuring at a constant temperature.

The maximum number of particles that can be determined is limited by the number of modes that can be measured. That is, the particle number is limited by the maximum measurable frequency and therefore depends on the scale of the cantilever. It has been found, that the position accuracy does not improve by using a higher number of modes than $N=2 M+1$.

Using the method proposed here it is possible to do quantitative single and multiple particle detection. It is thereby possible to do mass spectrometry on real samples where a low, but unknown number of particles adhere to the cantilever in between successive measurements. The method proposed can in principle be used to 
detect particles with different masses although it has not been demonstrated here.

\section{Acknowledgement}

This work has been financed by the EU FP7 NANODEVICE project.

\section{References}

[1] G. Y. Chen, T. Thundat, E. A. Wachter, R. J. Warmack, Journal of Applied Physics 77 (1995) 3618.

[2] T. Thundat, E. A. Wachter, S. L. Sharp, R. J. Warmack, Applied Physics Letters 66 (1995) 1695.

[3] B. Ilic, D. Czaplewski, M. Zalalutdinov, H. G. Craighead, P. Neuzil, C. Campagnolo, C. Batt, Journal of Vacuum Science \& Technology B 19 (2001) 2825 .

[4] D. Ramos, J. Mertens, M. Calleja, J. Tamayo, Sensors 7 (2007) 1757.

[5] M. Li, H. X. Tang, M. L. Roukes,Nature Nanotechnology 2 (2007) 114.

[6] A. K. Naik, M. S. Hanay, W. K. Hiebert, X. L. Feng, M. L. Roukes, Nature Nanotechnology 4 (2009) 445.

[7] E. Forsen, G. Abadal, S. Ghatnekar-Nilsson, J. Teva, J. Verd, R. Sandberg, W. Svendsen, F. Perez-Murano, J. Esteve, E. Figueras, et al.Applied Physics Letters 87 (2005) 043507. 
[8] S. Dohn, R. Sandberg, W. Svendsen, A. Boisen, Applied Physics Letters 86 (2005) 233501.

[9] H. T. Yu, X. X. Li, Applied Physics Letters 94 (2009) 011901.

[10] S. Dohn, W. Svendsen, A. Boisen, O. Hansen, Review of Scientific Instruments 78 (2007) 103303.

[11] J. C. Lagarias, J. A. Reeds, M. H. Wright, P. E. Wright, Siam Journal on Optimization 9 (1998) 112. 


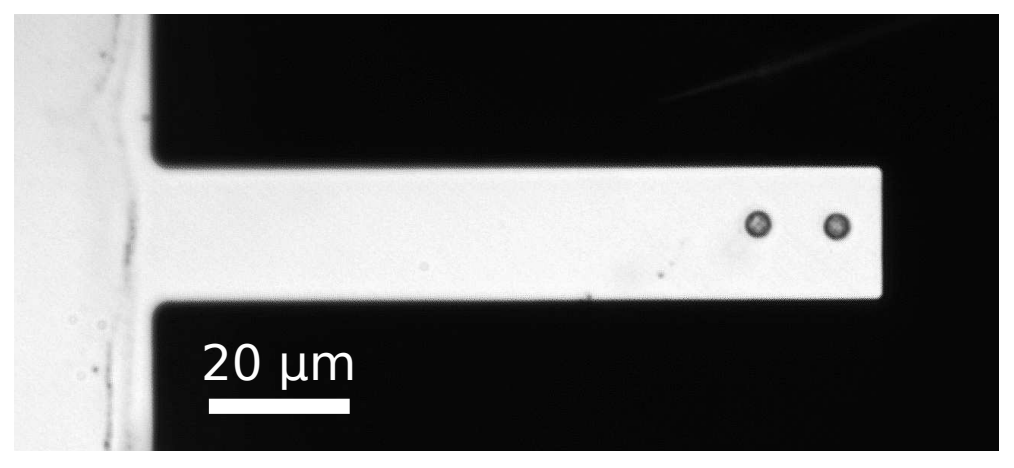

Fig. 1. Microscope image of a cantilever (D6) with two Dynabeads ${ }^{\circledR}$ attached. 


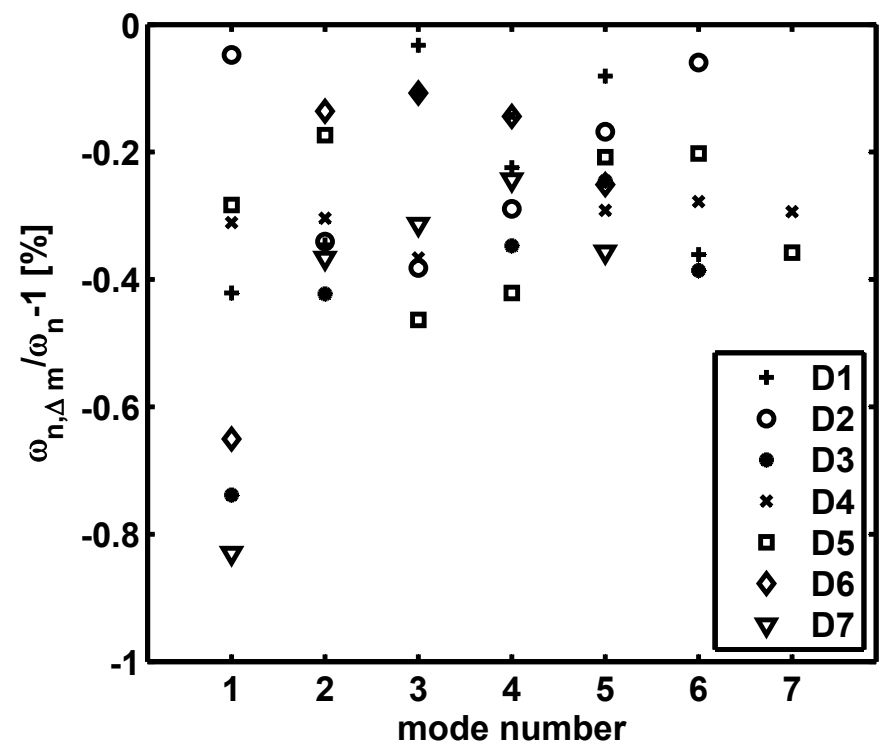

Fig. 2. Relative frequency shifts of cantilevers loaded with Dynabeads ${ }^{\circledR}$. 


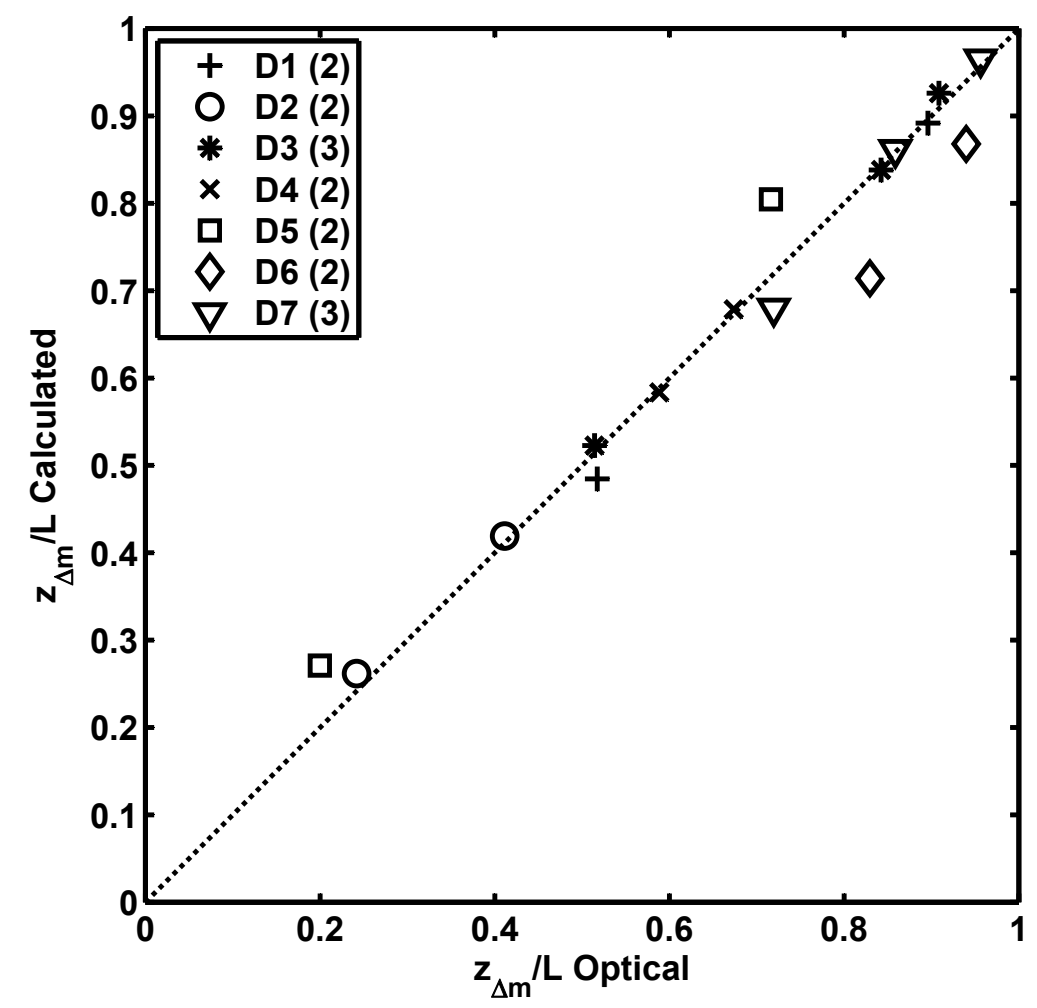

Fig. 3. Comparison of the Dynabeads ${ }^{\circledR}$ positions calculated from the measured resonant frequency shifts assuming all particles have different masses $(P=M)$ and the actual positions measured using an optical microscope. 